\title{
SYMMETRIC TENSORS OF THE SECOND ORDER WHOSE FIRST COVARIANT DERIVATIVES ARE ZER0*
}

BY

\author{
LUTHER PFAHLER EISENHART
}

1. Consider a Riemann space of the $n$th order, whose fundamental quadratic form, assumed to be positive definite, is written

$$
d s^{2}=g_{r s} d x^{r} d x^{s} \quad\left(g_{r s}=g_{s r}\right)
$$

where $r$ and $s$ are summed from 1 to $n$ in accordance with the usual convention which will be followed throughout this paper. It is well known that the first covariant derivatives $g_{r s / t}$ are zero, where

$$
g_{r s i t}=\frac{\partial g_{r s}}{\partial x^{t}}-g_{r a} \Gamma_{s}^{\alpha}-g_{a s} \Gamma_{r t}^{\alpha}
$$

and

$$
\Gamma_{s t}^{\alpha}=\frac{1}{2} g^{a p}\left(\frac{\partial g_{s p}}{\partial x^{t}}+\frac{\partial g_{t p}}{\partial x^{s}}-\frac{\partial g_{s t}}{\partial x^{p}}\right)
$$

the function $g^{a p}$ being the cofactor of $g_{a p}$ in the determinant

$$
g=\left|g_{r s}\right|
$$

divided by $g$. It is the purpose of this paper to determine the necessary and sufficient conditions that there exist a symmetric covariant tensor $\alpha_{r s}$ such that the first covariant derivatives $\alpha_{r s / t}$ are zero, or more than one such tensor.

2. Let $\alpha_{r s}$ denote the covariant components of any symmetric tensor of the second order. If $\varrho_{h}$ is a root of the equation

$$
\left|\alpha_{r s}-\rho g_{r s}\right|=0
$$

the functions $\lambda_{h}^{r}(r=1, \ldots, n)$ defined by

$$
\left(\alpha_{r s}-\varrho_{h} g_{r s}\right) \lambda_{h}^{r}=0
$$$$
(s=1, \ldots, n)
$$

* Presented to the Society, April 28, 1923. 
are the contravariant components of a vector. It is well known that the roots of (5) are real, and that if they are simple, the $n$ corresponding vectors at a point are mutually orthogonal. ${ }^{*}$ Moreover, if a root is of order $m$, equations(6) admit $m$ sets of independent solutions, and any linear combination of them is also a solution. It is possible to choose $m$ solutions so that the corresponding vectors at a point are mutually orthogonal, and thus from (6) obtain $n$ sets of solutions so that the corresponding vectors at a point are orthogonal; that is,

$$
g_{r s} \lambda_{h}^{r} \lambda_{k}^{s}=0 \quad(h, k=1, \ldots, n ; h \neq k) .
$$

Moreover, the components may be chosen so that

$$
g_{r s} \lambda_{h}^{r} \lambda_{h}^{s}=1 \quad(h=1, \ldots, n),
$$

that is, the vectors are unit vectors.

The curves in space whose direction at each point is defined by $\lambda_{h}^{r}$ form a congruence of curves $C_{h}$. Thus equations (6) define an $n$-uple of congruences of curves, such that the curves of the $n$-uple through a point are mutually orthogonal.

The covariant components $\lambda_{h, r}$ of the vector $h$ are given by

$$
\lambda_{h, r}=g_{r s} \lambda_{h}^{s}, \quad \lambda_{h}^{s}=g^{r s} \lambda_{h, r},
$$

and hence (7) and (8) are equivalent to

where

$$
\delta_{h k}=1 \text { for } h=k ;=0 \text { for } h \neq k .
$$

The functions $\gamma_{h i j}$ defined by

$$
\gamma_{h i j}=\lambda_{h, r / s} \lambda_{i}^{r} \lambda_{j}^{s}
$$

where $\lambda_{h, r / s}$ is the covariant derivative of $\lambda_{h, r}$ with respect to $x^{s}$, are invariants; they are called rotations by Ricci and Levi-Civita.t They have shown that

$$
\gamma_{h i j}+\gamma_{i h j}=0, \quad \gamma_{h h i}=0 \quad(h, i, j=1, \ldots, n) .
$$

* Cf. these Transactions, vol. 25 (1923), p. 259.

† Mathematische Annalen, vol.54 (1901), p.148; also, Wright, Invariants of Quadratic Differential Forms, Cambridge Tract, No. 9, p. 68. 
From (12) we have

$$
\lambda_{h, r / s}=\sum_{i, j}^{1} \dddot{\sum}_{h i j}^{n} \lambda_{i, r} \lambda_{j, s}
$$

and since $g_{r s / t}=0$, it follows from (9) that

$$
\lambda_{h / s}^{p}=\sum_{i, j}^{1} \gamma_{h i j} \lambda_{i}^{p} \lambda_{j, s}
$$

3. If all the roots of (5) are equal, we must have $\alpha_{r s}=\varrho g_{r s}$. Differentiating covariantly with respect to $x^{t}$, and making use of the fact that $g_{r s / t}=0$ and the assumption that $\alpha_{r s / t}=0$, we have that $\varrho$ is constant. Consequently $\alpha_{r s}$ is essentially the same as $g_{r s}$. We exclude this case from further consideration.

Since (7) is satisfied whether the functions $\lambda_{h}^{r}$ and $\lambda_{k}^{r}$ correspond to different simple roots of (5), or to the same multiple root when such exists, we have from (6)

$$
\alpha_{r s} \lambda_{h}^{r} \lambda_{k}^{s}=0 \quad(h, k=1, \ldots, n ; h \neq k) .
$$

Also from (6) we have

$$
\alpha_{r s} \lambda_{h}^{r} \lambda_{h}^{s}=\varrho_{h}
$$

From (17) we have by differentiating covariantly with respect to $x^{t}$ and making use of (15), (16), and (17)

$$
\alpha_{r s / t} \lambda_{h}^{r} \lambda_{h}^{s}=\frac{\partial \varrho_{h}}{\partial x^{t}}
$$

Also from (16) we have, because of (13), (14), (16) and (17),

$$
\alpha_{r s / t} \lambda_{h}^{r} \lambda_{k}^{s}+\sum_{j}^{1 \ldots n}\left(\varrho_{k}-e_{h}\right) \gamma_{h k j} \lambda_{j, t}=0
$$

Multiplying by $\lambda_{l}^{t}$ and summing for $t$, we have

$$
\alpha_{r s / t} \lambda_{h}^{r} \lambda_{k}^{s} \lambda_{l}^{t}+\left(\varrho_{k}-\varrho_{h}\right) \gamma_{h k l}=0 \quad(h \neq k) .
$$


From (18) it follows that if $\alpha_{r s / t}=0$ the roots $\varrho$ are constant. And from (19) we have for two different roots

$$
\gamma_{h k l}=0
$$

Let $\varrho_{1}$ be a root of (5) which we assume to be a multiple root of order $m$, and denote by $\lambda_{h}^{r}(h=1, \ldots, m)$ the components of the $m$ mutually orthogonal vectors corresponding to it, and by $\lambda_{k}^{r}(k=m+1, \ldots, n)$ the components of the directions corresponding to the other roots of (5). From (20) we have

$$
\gamma_{h k l}=0 \quad(h=1, \ldots, m ; k=m+1, \ldots, n ; l=1, \ldots, n) .
$$

Consider the system of equations

$$
X_{k}(f) \equiv \lambda_{k}^{r} \frac{\partial f}{\partial x^{r}}=0 \quad(k=m+1, \ldots, n) .
$$

If we introduce the notation

$$
\frac{\partial f}{\partial s^{k}}=\lambda_{k}^{r} \frac{\partial f}{\partial x^{r}}
$$

then, as Ricci and Levi-Civita have shown*, the relation

$$
\frac{\partial}{\partial s_{j}} \frac{\partial f}{\partial s_{k}}-\frac{\partial}{\partial s_{k}} \frac{\partial f}{\partial s_{j}}=\sum_{i}^{1 \ldots n}\left(\gamma_{i j k}-\gamma_{i k j}\right) \frac{\partial f}{\partial s_{i}}
$$

is satisfied for any function $f$.

Applying this formula to equations (22) we have in consequence of (21)

$$
X_{j} X_{k}(f)-X_{k} X_{j}(f) \stackrel{m+1 \ldots n}{=} \sum_{i}\left(\gamma_{i j k}-\gamma_{i k j}\right) X_{i}(f) \quad(j, k=m+1, \ldots, n) .
$$

Hence the system (22) is complete and admits $m$ independent solutions, say $f_{h}(h=1, \ldots, m)$.

\footnotetext{
* Loc. cit., p. 150; Wright, p. 69.
} 
Let $\varrho_{2}$ be another root of (5), of order $p$, and denote by $\lambda_{j}^{r}(j=m+1$, $\ldots, m+p)$ the components of the corresponding vectors. In like manner we show that the equations

$$
\lambda_{l}^{r} \frac{\partial f}{\partial x^{r}}=0 \quad(l=1, \ldots, m, m+p+1, \ldots, n)
$$

form a complete system and admit $p$ independent solutions $f_{j}(j=m+1$, ..., $m+p)$.

From (22) and the equations

$$
\lambda_{k}^{r} \lambda_{h, r}=0 \quad(h=1, \ldots, m ; k=m+1, \ldots, n)
$$

it follows that there exist functions $\alpha_{h}^{\sigma}$ such that

$$
\frac{\partial f_{h}}{\partial x^{r}}=\sum_{\sigma} a_{h}^{\sigma} \lambda_{\sigma, r} \quad(h, \sigma=1, \ldots, m) .
$$

In like manner, we have

$$
\frac{\partial f_{j}}{\partial x^{r}}=\sum_{\tau} b_{j}^{\tau} \lambda_{\tau, r} \quad(j, \tau=m+1, \ldots, m+p) .
$$

Consequently we have

$$
g^{r s} \frac{\partial f_{h}}{\partial x^{r}} \frac{\partial f_{j}}{\partial \dot{x}^{s}}=\sum_{\sigma, \tau} a_{h}^{\sigma} b_{j}^{\tau} g^{r s} \lambda_{\sigma, r} \lambda_{\tau, s}=0
$$

that is, any hypersurface $f_{h}=$ const. is orthogonal to each of the hypersurfaces $f_{j}=$ const.

Proceeding in this manner with the other roots of (5) we obtain a group of hypersurfaces corresponding to each distinct root of (5), the number of hypersurfaces in a group being equal to the order of the root. Any two hypersurfaces of two different groups are orthogonal to one annther. If we take these $n$ families of hypersurfaces for the parametric surfaces $x^{r}=$ const. $(r=1, \ldots, n)$, it follows that the functions $g_{r s}$ are zero, for the case where $x^{r}=$ const. and $x^{s}=$ const. are hypersurfaces of different groups; in this sense we say that $r$ and $s$ refer to different groups, or different roots of (5). From the equations (22) for this choice of the variables $x$, it follows that $\lambda_{k}^{r}=0$, for $r$ and $k$ referring to different roots of (5). From (9) it follows also that $\lambda_{k, r}=0$ for $k$ and $r$ referring to different roots. 
Equations (6) may be replaced by*

$$
\alpha_{r s}=\sum_{h}^{1 \ldots n} \rho_{h} \lambda_{h, r} \lambda_{h, s}
$$

whether the roots of (5) are simple, or some are multiple. From (24) and the preceding observations it follows

$$
\begin{aligned}
& \alpha_{r s^{\prime}}=g_{r s^{\prime}}=0, \\
& \alpha_{r s}=\rho_{h} g_{r s},
\end{aligned}
$$

where $r$ and $s^{\prime}$ refer to any two different roots and $r$ and $s$ refer to the root $\varrho_{h} . t$

4. From (25) we have $\alpha_{r s^{\prime}}=0$, hence if $\alpha_{r s^{\prime} / t}=0$, we must have (cf. (2))

$$
\alpha_{r l} \Gamma_{s^{\prime} t}^{l}+\alpha_{s^{\prime} q} \Gamma_{r t}^{q}=0 \quad(l, q=1, \ldots, n)
$$

that is

$$
\alpha_{r l} g^{l \beta}\left[\frac{\partial g_{s^{\prime} \beta}}{\partial x^{t}}+\frac{\partial g_{t \beta}}{\partial x^{s^{\prime}}}-\frac{\partial g_{s^{\prime} t}}{\partial x^{\beta}}\right]+\alpha_{s^{\prime} q} g^{q \beta}\left[\frac{\partial g_{r \beta}}{\partial x^{t}}+\frac{\partial g_{t \beta}}{\partial x^{r}}-\frac{\partial g_{r t}}{\partial x^{\beta}}\right]=0
$$

If $r$ refers to the root $\varrho_{1}$ of (5), say $r=1, \ldots, m$ and $s^{\prime}$ to the root $\varrho_{2}$, say $s^{\prime}=m+1, \ldots, m+p$, we have from (25)

$$
\begin{array}{lr}
\alpha_{r l}=\varrho_{1} g_{r l} & (l=1, \ldots, m) ; \\
\alpha_{r l}=0 & (l=m+1, \ldots, n) ; \\
\alpha_{s^{\prime} q}=\varrho_{2} g_{s^{\prime} q} & (q=m+1, \ldots, m+p) ; \\
\alpha_{s^{\prime} q}=0 & (q=1, \ldots, m, m+p+1, \ldots, n) .
\end{array}
$$

Hence the above equation reduces to

$$
\varrho_{1}\left(\frac{\partial g_{s^{\prime} r}}{\partial x^{t}}+\frac{\partial g_{t r}}{\partial x^{\prime}}-\frac{\partial g_{s^{\prime} t}}{\partial x^{r}}\right)+\varrho_{s}\left(\frac{\partial g_{r s^{\prime}}}{\partial x^{t}}+\frac{\partial g_{t s^{\prime}}}{\partial x^{r}}-\frac{\partial g_{r t}}{\partial x^{s^{\prime}}}\right)=0 .
$$

* Cf. Ricci and Levi-Civita, loc. cit., p. 159.

† Cf. Levi-Civita, Annalioli Matematica, ser. 2, vol. 24 (1896), p. 298. 
If now $t$ and $v$ refer to the same root, this equation reduces to

$$
\left(\rho_{1}-\rho_{2}\right) \frac{\partial g_{t r}}{\partial x^{\prime}}=0
$$

and if $t$ and $s^{\prime}$ refer to the same root, we have

$$
\left(\varrho_{1}-\varrho_{2}\right) \frac{\partial g_{s} t^{\prime}}{\partial x^{r}}=0
$$

If $r, s^{\prime}$ and $t$ refer to three different roots, the equation vanishes identically. Since $\rho_{1}$ and $\rho_{2}$ are not equal by hypothesis, we have that each function $g_{r \cdot s}$ depends only on the coordinates referring to the same root as $r$ and $s$.

Consider again

$$
\alpha_{r s}=\rho_{1} g_{r s} \quad(r, s=1, \ldots, m) .
$$

Now

$$
\alpha_{r s / t}=\rho_{1} \frac{\partial g_{r s}}{\partial x^{t}}-\alpha_{r l} \Gamma_{s t}^{l}-\alpha_{s l} \Gamma_{r t}^{l} \quad(l=1, \ldots, n)
$$

which by (25) is reducible to

$$
\alpha_{r s ! t}=\varrho_{1}\left(\frac{\partial y_{r s}}{\partial x^{t}}-g_{r l} \Gamma_{s t}^{l}-g_{s l} \Gamma_{r t}^{l}\right)=\varrho_{1} g_{r s, t}=0
$$

Hence we have the following theorem:

$A$ necessary and sufficient condition that a Riemann space admit a symmetric covariant tensor of the second order $\alpha_{r \cdot s}$ other than, with a positive definite fundamental form (1), $g_{r s}$, such that its first covariant derivative is zero, is that (1) be reducible to a sum of forms

$$
\varphi^{(i)}=g_{r_{i} s_{i}}^{(i)} d x^{r_{i}} d x^{s^{i}}
$$

where $g_{r_{i} s_{i}}^{(i)}$ are functions at most of the $x$ 's of that form; then

$$
\boldsymbol{\alpha}_{r s} d x^{r} d x^{s}=\sum_{i} \rho_{i} \varphi^{(i)}
$$

where the $\rho^{\prime} s$ are arbitrary constants. 
In particular, if all the roots of (5) are simple, the space is euclidean; if its fundamental form is taken in the form

$$
d s^{2}=\sum_{i} d x^{i^{*}} \quad(i=1, \ldots, n),
$$

then

$$
\boldsymbol{\alpha}_{r \cdot s} d x^{r} d x^{s}=\sum_{i} \varrho_{i} d x^{i^{\boldsymbol{n}}} \quad(i=1, \ldots, n)
$$

where the $\rho$ 's are $n$ different arbitrary constants.

When any one of the roots of (J) is simple, the corresponding congruence is normal, and the tangents to the congruence form a field of parallel vectors in the sense of Levi-Civita.*

5. In this section it will be shown that the problem of determining whether a given Riemann space admits one, or more, symmetric tensors whose first covariant derivatives are zero is a problem of algebra.t

We recall that if $\alpha_{r \cdot s}$ is any symmetric tensor, then

$$
\alpha_{r \cdot s / j k}-\alpha_{r s / k j}=\alpha_{r t} B_{s j k}^{t}+\alpha_{s t} B_{r j k}^{t}
$$

where $\alpha_{r s / j k}$ is the second covariant derivative of $\alpha_{r s}$, and $B_{s j k}^{t}$ are the components of the Riemann tensor of the second kind formed with respect to (1). If then $\alpha_{r \cdot s / j}=0$, we must have

$$
\alpha_{r s / j k}-\alpha_{r s / k j}=0
$$

and consequently we have equations of the form

$$
\boldsymbol{\alpha}_{r t} B_{s j k}^{t}+\alpha_{s t} B_{r j k}^{t}=0 \quad(\jmath, k, r, s, t=1, \ldots, n) .
$$

Differentiating these equations covariantly successively we have the sets of equations

$$
\begin{aligned}
& \alpha_{r t} B_{s j k / m_{1}}^{t}+\alpha_{s t} B_{r j k / m_{1}}^{t}=0, \\
& \alpha_{r t} B_{s j k / m_{1} \ldots m_{l}}^{t}+\alpha_{s t} B_{r j k / m_{1} \ldots m_{l}}^{t}=0 \text {, }
\end{aligned}
$$

* Cf. Proceedings of the National Academy of Sciences, vol. 8 (1922), p. 211.

fCf. Eisenhart and Veblen, Proceedings of the National Academy of Sciences, vol. 8 (1922), p. 23; also Veblen and Thomas, these Transactions, vol. 25 (1923). 
Since $g_{r s}$ satisfies (28) the systems (29) and (30) are satisfied by $g_{r s}$ and consequently are algebraically consistent. From this it follows either that the functions $g_{r s}$ are the only solution of (29) and (30), or that (29) and the first $l(\geqq 0)$ sets of (30) admit a complete system of solutions $g_{r s}$ and $\alpha_{r s}^{(1)}, \ldots, \alpha_{r s}^{(p)}$ which satisfy also the $(l+1)$ th set of equations $(30)$. In the latter case the general solution is of the form

$$
\boldsymbol{\alpha}_{r s}=\varphi^{(0)} a_{r s}+\varphi^{(1)} \boldsymbol{\alpha}_{r s}^{(1)}+\cdots+\varphi^{(p)} \boldsymbol{\alpha}_{r s}^{(p)} .
$$

If any one of the functions $\alpha_{r s}^{(\sigma)}(\sigma=1, \ldots, p)$ is substituted in (29) and the first $l$ sets of (30), and these equations are differentiated covariantly, we have, in consequence of the above requirement, that the functions $\alpha_{r s / m}^{(\sigma)}$ $(\sigma=1, \ldots, p ; m=1, \ldots, n)$ satisfy $(29)$ and the first $l$ sets of $(30)$. Consequently we have

$$
\boldsymbol{\alpha}_{r s / m}^{(\boldsymbol{\sigma})}=\lambda_{m}^{(\boldsymbol{\sigma}))} g_{r s}+\lambda_{m}^{(\boldsymbol{\sigma})} \boldsymbol{\alpha}_{r s}^{(1)}+\cdots+\lambda_{m}^{(\sigma p)} \boldsymbol{\alpha}_{r s}^{(p)},
$$

where the $p(p+1)$ vectors $\lambda_{m}^{(\sigma \beta)}(\sigma=1, \ldots, p ; \beta=0,1, \ldots, p)$ must be such that the functions (32) shall satisfy (28). Substituting in these equations we find that the functions $\lambda$ must satisfy the system

$$
\frac{\partial \lambda_{p}^{(\sigma \tau)}}{\partial x^{q}}-\frac{\partial \lambda_{q}^{(\sigma \tau)}}{\partial x^{p}}+\sum_{\omega}\left(\lambda_{p}^{(r \omega)} \lambda_{q}^{(\omega \tau)}-\lambda_{q}^{(\sigma \omega)} \lambda_{p}^{(\omega \tau)}\right)=0\left(\begin{array}{l}
\sigma, \omega=1, \ldots, p \\
\tau=0,1, \ldots, p
\end{array}\right)
$$

In order that $\alpha_{r s}$ given by (31) shall satisfy $\alpha_{r s / t}=0$, it is necessary and sufficient that the functions $\varphi^{(i)}$ satisfy

$$
\frac{\partial \varphi^{(0)}}{\partial x^{t}}+\sum_{\sigma} \varphi^{(\sigma)} \lambda_{t}^{(\sigma 0)}=0 \quad(\sigma=1, \ldots, p)
$$

and

$$
\frac{\partial \varphi^{(\tau)}}{\partial x^{t}}+\sum_{\sigma} \varphi^{(\sigma)} \lambda_{t}^{(\sigma \tau)}=0 \quad(\sigma, \tau=1, \ldots, p)
$$

In consequence of (33) equations (35) are completely integrable and therefore admit solutions involving $p$ arbitrary constants. Because of (33) the conditions of integrability of (34) are satisfied; hence $\varphi^{(0)}$ involves these $p$ arbitrary constants and an additive arbitrary constant which may be neglected.*

* If $\alpha_{r e}$ is a tensor whose first covariant derivative 1s zero, so also is $\alpha_{r s}+\lambda g_{r e}$, where $\lambda$ is an arbitrary constant. 
In view of the above results we have the theorem:

If equations (29) and the first $l(\geqq 0)$ sets of equations (30) admit a complete system of solutions $g_{r s}$ and $\alpha_{r s}^{(\sigma)}(\sigma=1, \ldots, p)$ which are also solutions of the $(l+1)$ th set of equations (30), there exists a symmetric tensor of the second. order, involving $p$ arbitrary constants, whose first covariant derivative is zero.

6. Suppose that the fundamental form is the sum of $j$ forms (26). By definition

$$
B_{p r s}^{\alpha}=g^{a q} B_{p q r s}
$$

where $B_{p q r s}$ is the covariant Riemann tensor of the fourth order, that is,

$$
B_{p q r s}=\frac{1}{2}\left(\frac{\partial^{2} g_{p s}}{\partial x^{q} \partial x^{r}}+\frac{\partial^{2} g_{q r}}{\partial x^{p} \partial x^{s}}-\frac{\partial^{2} g_{p r}}{\partial x^{q} \partial x^{s}}-\frac{\partial^{2} g_{q s}}{\partial x^{p} \partial x^{r}}\right)
$$

where

$$
+g^{l m}\left(\boldsymbol{\Gamma}_{p s, m} \Gamma_{q r, l}-\boldsymbol{\Gamma}_{p r, m} \boldsymbol{\Gamma}_{q s, l}\right),
$$

For the case under consideration, namely (26), it is readily shown that the components $B_{p q r s}$ are zero, unless $p, q, r, s$ refer to the same root of (5); likewise $B_{p r s, s}^{\alpha}$, and its first covariant derivatives $B_{p r s / t}^{\alpha}$. Consequently equations (29) and the first set of (30) admit, in addition to $g_{r s}$, the $j$ sets of solutions of the form (25). If it is understood that each of the forms (26) is not further reducible to sums of such forms, we have a complete set of solutions of (29). Hence when the space is referred to the corrdinates giving (25) the number $l$. in the preceding theorem is zero.

PRINCETON UNIVERSITY,

Princeton, N. J. 